\title{
Assessment of autonomic dysfunction between type I and type II diabetes mellitus
}

\author{
Kailash S Mottera ${ }^{1}$, Shiva Kumar ${ }^{2 *}$ \\ ${ }^{1,2}$ Consultant Physician, Dept. of Internal Medicine, ${ }^{1}$ Lotus Hospitals, HSR Layout, Bangalore, Karnataka, ${ }^{2}$ Suryodaya Healthcare, Malur, \\ Karnataka, India \\ *Corresponding Author: Shiva Kumar \\ Email: drshivkr@gmail.com
}

\begin{abstract}
Introduction: The diabetes is the most common disease in the present world and India is country with highest incidence of Diabetes Mellitus in the world. The autonomic nervous dysfunction due to diabetes is the common complication of the diabetes. In this study autonomic dysfunction in pateints with type I and type $2 \mathrm{DM}$ and its correlation with the duration of diseases is studied.

Materials and Methods: The study was conducted on 30 type I diabetic patients and 40 type II diabetic pateints. All the diabetic patients were questioned about the presence of symptoms reported to be related to autonomic neuropathy. The conventional autonomic function tests were performed on all the subjects inclusive of sympathetic and parasympathetic tests. The scoring of positive and negative for autonmomic dysfuntion was done.

Results: The duration of disease in type 1 diabetes, was 6 to 15 years and in type 2 diabetes it was 8 to 18 years with mean duration of 8.5 and 14 years respectively. The mean HbA1C in type $1 \mathrm{DM}$ was $6.21 \pm 2.09$ and it was $7.50 \pm 2.45$ in type 2 DM. Comparison of autonomic function tests in the form of heart rate and BP response in subjects with type 1 and type 2 showed that there no significant statistical difference between the groups.

Conclusion: It can be concluded the presence of autonomic dysfunction in type 1 and type 2 diabetes mellitus might be due to nerve damage. The duration of diabetes is directly related to such autonomic dysfunction.
\end{abstract}

Keywords: Diabetes Mellitus type 1, Diabetes Mellitus type 2, Autonomic Functions Tests.

\section{Introduction}

The word "Diabetes" came from a Greek word meaning, "to run through". Thomas Willis observed that urine of diabetes was "wonderfully sweet" and Dobson (1775) demonstrated that the sweetness was due to sugar. In 1869, Langerhans discovered the islets of pancreas, which was later given his name. In 1889 a great landmark was reached when Von Mering and Minowski produced diabetes in dogs by total pancreatomy.

The diabetes is the most common disease in the present world and India is country with highest incidence of Diabetes Mellitus in the world. ${ }^{1}$ Prevalence of diabetes in adults in India was found to be $2.4 \%$ in rural and $4-11.6 \%$ in urban dwellers. High frequencies of impaired glucose tolerance, shown by those studies, ranging from 3.6-9.1\% indicate the potential for further rise of prevalence of diabetes in the coming decades. ${ }^{2}$

The types of diabetes mellitus are Type 1 Diabetes: which occurs because of the beta cell destruction, usually leading to absolute insulin deficiency, it can be immune mediated or idiopathic. Type 2 diabetes: may range from predominantly insulin resistance, with relative insulin deficiency, to a predominantly secretory defect with insulin resistance. Other specific diabetes includes gestational diabetes mellitus, genetic defects, and diseases of pancreas, drug induced and diabetes occuring in syndromes. ${ }^{3}$

There are several complications of diabetes mellitus and it involves several organs of the body. The autonomic nervous dysfunction due to diabetes is the common complication of the diabetes. Diabetic Autonomic Neuropathy can be classified on the basis of presence or absence of symptoms or the nerve or organ involved. Overt symptomatic and/or subclinical - elicited by detailed diagnostic tests, parasympathetic/sympathetic/ sdrenomedullary. ${ }^{4}$ It can also be classified on the basis of organs involved cardiovascular system, ocular system, gastrointestinal system, sudomotor and endocrine.

Autonomic nervous system consists of the sympathetic and parasympathetic systems. There are 2 main efferent limbs of the autonomic nervous system. Post-ganglionic parasympathetic fibers from the vagus innervate cardiac, pulmonary and upper gastrointestinal organs, while the pelvic organs are innervated by the sacral parasympathetic outflow. A sympathetic innervation is derived from the preganglionic fibers whose cell bodies are in the interomediolateral column of the spinal cord at the level of the thoracic and upper lumbar roots. These nerve synapses in the bilateral chain of the sympathetic ganglia with postganglionic neurons which widely innervate the vascular smooth muscles, heart kidney, gut and other organs.

It is observed that the autonomic dysfunction in diabetes has significanctly contribute to $50 \%$ of 5 -year mortality, Sudden death common (consider electrophysiological studies), Greater complications after elective surgery, Increased danger with general anaesthesia, the significant increase in major microvascular complications makes it important to screen diabetes at a younger age of 45 years. ${ }^{5,6}$

The frequency of diabetic autonomic neuropathy is difficult to ascertain reliably. Between $17 \& 40 \%$ of conservative or randomly selected adult diabetics have abnormal cardiovascular function tests according to most large series. $31 \%$ of a group of teenage diabetics and $15 \%$ of a group of younger diabetic children had abnormal cardiovascular reflexes symptoms usually occur after a prolonged duration. Pathogenesis is similar to that of 
somatic neuropathy and is suggested by a generalized correlation between the two. ${ }^{7,8}$

Autonomic neuropathy was only generally recognized as part of the spectrum of nerve damage in diabetes. By the time symptoms have developed, autonomic nerve damage is probably irreversible and carries a poor prognosis. As some autonomic damage occurs in many diabetics, however, prevention of the late stages is clearly desirable. ${ }^{9,10}$

In this study autonomic dysfunction in pateints with type I and type $2 \mathrm{DM}$ and its correlation with the duration of diseases is studied.

\section{Materials and Methods}

The study was conducted in Adichunchinagiri Institute of Medical Sciences in the Department of Medicine. The study was conducted on 30 type I diabetic patients and 40 type II diabetic pateints.

\section{Inclusion criteria}

\section{Criteria for diagnosis of Diabetes Type 2}

Symptoms of Diabetes (polyuria, polydipsia, polyphagia, increased fatigue, weight loss, blurred vision, growth impairment) with Random Blood Glucose (venous blood) concentration of $200 \mathrm{mg} / \mathrm{dl}$ or more or fasting (of more than 8 hours) blood glucose levels of $126 \mathrm{mg} / \mathrm{dl}$ or more or twohour Post-prandial blood glucose levels of $200 \mathrm{mg} / \mathrm{dl}$ or more.

Criteria for diagnosis of Diabetes Type 1: Subjects with age less than 30 years, and history of symptoms of diabetes were included in study.

The following tests were performed to assess the autonomic functions in the above patients. The tests reflecting parasympathetic function are heart rate variation during deep breathing, heart rate response to valsalva maneuver, immediate heart rate response to standing. The tests reflecting sympathetic functions were blood pressure response to standing and blood pressure response to sustained handgrip. The test procedure was conducted as per the standard protocol as described by D J Ewing et al. ${ }^{12}$

Intepretation of the tests is done by calculating the scores according to Ewing's criteria. ${ }^{11,12}$ Ewing's, the scores are calculating as normal, borderline and abnormal values.

\section{Results}

The average age of the particpants included in the study is $34.18 \pm 12.56$ years for type 2 diabetes and $45.36 \pm 15.07$ years for type 1 diabetes. The range was from 18 to 48 years and 24 to 54 years in subjects with type 1 and type 2 diabetes respectively. Maxiumum number of pateints was seen between 25 to 30 years among type 1 diabetes group and between 40-45 years in type 2 diabetic group.

The duration of disease in type 1 diabetes, was 6 to 15 years and in type 2 diabetes it was 8 to 18 years with mean duration of 8.5 and 14 years respectively. Significant proportion patients were in 6-10 years duration. The comparison of FBS and PPBS between type 1 and type 2 diabetes patients are presented in the table 1. The comparison of HbAlc between type 1 and type 2 diabetes patients are presented in the Table 1 and Fig. 1.

Table 1: Comparison of FBS, PPBS and HBa1c between Type 1 and Type 2 patients

\begin{tabular}{|l|c|c|c|}
\hline & Type 1 & Type 2 & P Value \\
\hline FBS $(\mathrm{mg} / \mathrm{dl})$ & $131.90 \pm 35.74$ & $123.93 \pm 35.92$ & 0.360 \\
\hline PPBS $(\mathrm{mg} / \mathrm{dl})$ & $226.50 \pm 57.58$ & $207.70 \pm 57.93$ & 0.182 \\
\hline HbA1C & $6.21 \pm 2.09$ & $7.50 \pm 2.45$ & $0.023^{*}$ \\
\hline
\end{tabular}

$* \mathrm{P}<0.05$ is considered statistical significant

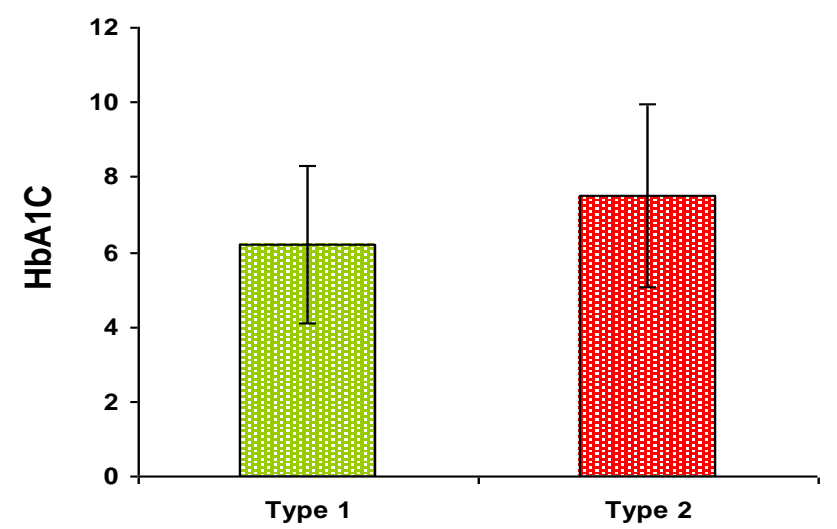

Fig. 1: Comparison HBa1c between Type 1 and Type 2 patients in cases

Ewing's Autonomic test scoring system is used to evaluate if a patient had autonomic dysfuntion. This system is described in the methods. It has maximum total score of 10 to a minimum of 0 , a score of more than 5 , i.e. 6 or more was considered as positive autonomic scores. The distribution of autonomic dysfunction score was positive in 19 patients with type 1 and 25 patients with type 2 had positive autonomic scores (Table 2). Chi square test was applied between the two groups and there is statistical significance between the type 1 and type 2 diabetes mellitus.

Table 2: Distribution of Autonomic scores among the type 1 and type 2 DM

\begin{tabular}{|l|c|c|c|c|c|}
\hline \multirow{2}{*}{ Autonomic score } & \multicolumn{2}{|c|}{ Type I DM } & \multicolumn{2}{c|}{ Type II DM } & \multirow{2}{*}{ P value } \\
\cline { 2 - 5 } & n & Percentage & n & Percentage & \\
\hline Negative $(<=5.0)$ & 11 & 36.6 & 15 & 37.5 & 0.939 \\
\hline Positive $(>5.0)$ & 19 & 64.4 & 25 & 62.5 & 0.871 \\
\hline
\end{tabular}


Table 3: Comparison of Heart rate and BP response in two groups of patients

\begin{tabular}{|l|c|c|c|}
\hline & Type 1 DM & Type 2 DM & P Value \\
\hline Heart rate response to deep breathing & $12.26 \pm 4.95$ & $14.04 \pm 5.26$ & 0.189 \\
\hline Heart rate response to valsalva maneuver & $1.07 \pm 0.27$ & $1.18 \pm 0.19$ & 0.051 \\
\hline Immediate heart rate response to standing & $1.01 \pm 0.15$ & $1.07 \pm 0.19$ & 0.158 \\
\hline B.P response to standing & $14.88 \pm 7.51$ & $10.96 \pm 9.31$ & 0.063 \\
\hline B.P response to handgrip & $14.40 \pm 5.57$ & $12.24 \pm 3.95$ & 0.061 \\
\hline
\end{tabular}

Comparison of heart rate and BP response in subjects with type 1 and type 2 is presented in the table 3 . There is no significant statistical difference in the values between the groups (Student $t$ test). The comparison of autonomic scores and study variables was done using the unpaired $t$ test. Significant difference between participants with positive autonomic scores and negative autonomic scores in the study participants. (Table 4).

Table 4: Comparison of study variables in Autonomic score positive and negative score among the study participants.

\begin{tabular}{|l|c|c|c|}
\hline \multirow{2}{*}{ Variables in diabetics cases } & \multicolumn{2}{|c|}{ Autonomic score } & \multirow{2}{*}{ P value } \\
\cline { 2 - 3 } & Negative & Positive & \\
\hline Age in years & $42.91 \pm 12.59$ & $54.43 \pm 15.12$ & $0.001^{* *}$ \\
\hline Duration of diabetics in years & $5.39 \pm 3.06$ & $10.96 \pm 6.95$ & $0.001^{* *}$ \\
\hline Heart rate variation in deep breathing & $16.41 \pm 3.11$ & $9.00 \pm 3.45$ & $<0.001^{* *}$ \\
\hline Heart rate variation in Valsalva & $1.27 \pm 0.13$ & $0.91 \pm 0.25$ & $<0.001^{* *}$ \\
\hline Heart rate variation in standing & $1.11 \pm 0.06$ & $0.93 \pm 0.16$ & $<0.001^{* *}$ \\
\hline B.P. variation on standing & $12.18 \pm 4.69$ & $17.00 \pm 8.63$ & $0.023^{*}$ \\
\hline B.P. variation on handgrip & $17.09 \pm 4.44$ & $12.29 \pm 5.52$ & $0.002^{*}$ \\
\hline Glycosylated Haemoglobin & $5.98 \pm 1.41$ & $8.24 \pm 2.59$ & $0.001^{* *}$ \\
\hline
\end{tabular}

$* \mathrm{P}<0.05$ is considered statistical significant, $* * \mathrm{P}<0.001$ is considered high statistical significant,

Table 5: Common presenting symptoms common in type 1 and type $2 \mathrm{DM}$

\begin{tabular}{|l|c|c|}
\hline \multicolumn{1}{|c|}{ Symptoms } & Type 1 ( out of 30) & Type 2 (out of 40) \\
\hline Impotence & 10 & 17 \\
\hline Postural hypotension & 4 & 11 \\
\hline Constipation & 6 & 10 \\
\hline Diarrhea & 2 & 6 \\
\hline Bladder disturbances & 8 & 14 \\
\hline Ulcers on foot & 6 & 12 \\
\hline
\end{tabular}

The presenting complaints were in the form of symptoms like impotence (most common), postural hypotension, constipation, diarrhea, bladder disturbances, ulcers on the foot. Other complaints were visual distubances, urinary tract infections.

\section{Discussion}

The incidence of autonomic dysfunctions increased with the increasing duration of diabetes.Maxiumum number of pateints was seen between 25 to 30 years among type 1 diabetes group and between $40-45$ years in type 2 diabetic groups. Dyrberg et $\mathrm{al}^{13}$ reported an incidence of $15 \%$ autonomic neuropathy in diabetics of duration up to 10 years and $62 \%$ in diabetics of more than 10 years. M.Lakhotia, S.S. Jain, et al., $1997^{14}$ showed a great incidence of dysautonomia with increasing duration (up to $80 \%$ in those with duration of more than 5 years).

The duration of disease in type 1 diabetes, was 6 to 15 years and in type 2 diabetes it was 8 to 18 years with mean duration of 8.5 and 14 years respectively. This indicates that there is correlation between the duration of diabetes and the autonomic dysfunction as seen in both type 1 and type 2 diabetes mellitus.

Autonomic dysfunctions are also associated with poor glycemic control as seen by the mean HbAlc values in type 1 and type 2 diabetes mellitus. The mean value of glycosylated haemoglobin was $6.21 \pm 2.09 \%$ among Type 1 patients. It was $7.50 \pm 2.45$ among Type 2 diabetic patients. The target HbA1c in normal individuals is $7.0 \%$ in a study by R.C. Gupta, et al., ${ }^{15}$ after 6 months of strict metabolic control they found that $22 \%$ patients showed significant symptomatic improvement.

It is seen that the there are several mechanisms by which the autonomic dysfunction occurs in both type 1 and type 2 diabetes mellitus. One of the mechanisms is vasular presence of arterial stiffness because of vascular denervation which causes structural and functional changes in arterial smooth muscle leading to calcification and ossification. Endoneural capillaries of patients with diabetic neuropathy exhibit an increased endothelial cell proliferation and capillary closure that correlates with the seventy of the neuropathy. Segmental loss of myelinated fibers, seen in both peripheral and splanchnic nerves, may represent areas 
of regional ischemia caused by closure of provider capillaries, fiber loss increases from proximal to distal nerve, reflecting recurrent areas of proximal ischemia resulting in profound distal nerve dysfunction. Endoneural blood flow is one third of that in healthy state. Endoneural oxygen tension is decreased. ${ }^{16,17}$

In diabetes mellitus, the vascular endothelium often produces and releases abnormally low amounts of plasminogen activator, leading to an impaired fibrinolytic system, which might be of importance for the development of angiopathy. The desaturation reactions and especially the 6 desaturation pathway are impaired and there is deficient conversion of linoleic acid to gamma linoleic acid, despite normal dietary intake of essential fatty acids. These leads to abnormal cell membrane, membrane bound enzymes and receptors and myelin turnover, resulting in decreased nerve conduction velocities. Further more, the endoneural hypoxia suppresses ATPase activity, promoting paranodal demyelination as well as diminished axonal transport (Axonopathy). ${ }^{18}$

Disturbances in delta-6-desaturase in the n-6 pathway of essential fatty acids lead to reduced formation of gamma linoleic acid, di-homo gamma linoleic acid and arachidonic acid (precursors of prostaglandin). Deficiency of prostacyclin with increase in formation of thromboxane A2 impairs the microcirculation of the vasa vasorum, leading to endoneural hypoxia and a vicious cycle of more capillary damage and further hypoxia. The release of oxygen free radicals further damages endothelial cell functions; axonopathy and myelinopathy dislocate axonal transport by direct impairment of neural ATPase activity. This is reflected by impaired nerve conduction velocity. ${ }^{19}$

\section{The metabolic abnormalites that is noted are myoinositol deficiency ${ }^{20}$}

Myoinositol is a normal dietary cyclic hexose, structurally similar to glucose, concentrated about hundred times more in the nerves than in plasma. When membrane receptor is stimulated, myoinositol liberates second messengers, inositol triphosphate and diacylglycerate, which act to release intra cellular calcium and activate protein kinase. This mediates ATP utilisation through sodium potassium ATPase activity. Hyperglycemia results in competitive inhibition of sodium myoinositol uptake system causing a low myoinositol level and hence poor sodium ATPase. This decreases nerve cell membrane potential. Hence the conduction velocity decreases, and also lowers further the myoinositol uptake, setting up a vicious cycle. Sorbitol accumulation high glucose concentration stimulates aldose reductase and promotes polyol pathways, thus, more and more glucose is converted to sorbitol, which in turn is metabolized to fructose. Rising sorbitol levels play an unclear role in the pathogenesis of diabetic neuropathy. Aldose reductase inhibitors have beneficial effects in treating as well as preventing autonomic neuropathy. Non enzymatic glycosylation similar to that seen in a RBC, hemoglobin results in aggregation of tubulin and microtubulin affecting cellular transport profoundly. This might represent the pathophysiologic mechanism of nerve damage..$^{21-23}$

It can be concluded the presence of autonomic dysfunction in type 1 and type 2 diabetes mellitus might be due to nerve damage. The duration of diabetes is directly related to such autonomic dysfunction. Strict glycemic control might reduce the autonomic dysfunction in diabetes. It also suggested that pateints should be encouraged to undergo autonomic functions tests for any early detection of such symptoms.

\section{Source of funding}

None.

\section{Conflict of interest}

None.

\section{References}

1. Michael Brownlee, Lloyd. P. Aiello, Mark.E.Cooper, William's textbook of Endocrinology, $11^{\text {th }}$ edition, chapter 32, 1418-1490

2. K. Park. "Diabetes Mellitus", Park's textbook of Preventive and Social Medicine, $20^{\text {th }}$ edition, pgs 341-343

3. Gabir MM, Hanson RL, Dabela D. The 1997 American Diabetic Association and 1999 World Health Organization criteria for Hyperglycemia in the diagnosis and prediction of Diabetes. Diabetes Care 2000;23:1108-12.

4. Noronha JL, Bhandarkar SD, Shenoy PN, Retnam VJ. Autonomic Neuropathy in Diabetes Mellitus. J Postgrad Med 1981;27:1-6.

5. Raelene E. Maser and M. James Lenhard. REVIEW: Cardiovascular Autonomic Neuropathy Due to Diabetes Mellitus: Clinical Manifestations, Consequences, and Treatment. J Clin Endocrinol Metab 90(10):5896-5903.

6. Carolina M. Casellini, Aaron I. Vinik. Clinical Manifestations and Current Treatment Options for Diabetic Neuropathies. Endocr Pract 2007;13(5):550-66.

7. Watkins PJ. Progression of diabetic autonomic neuropathy. Diabetic Med 1993;(10):775-875.

8. D J Ewing, B F Clarke. Diagnosis and management of diabetic autonomic neuropathy. Br Med J 1982;285:916-8.

9. Sainani G.S. Current concepts in diabetes mellitus, $1^{\text {st }}$ edition. Pgs 48-65, 1993.

10. Johnson P.C. Pathogenesis of diabetic neuropathy. Ann Neurol 1986;19:450-7.

11. D J Ewing, B F Clarke. Diagnosis and management of diabetic autonomic neuropathy. Br Med J 1982;285:916-8.

12. D J. The value of cardiovascular autonomic function tests, 10 years experience in diabetes. Diabetes Care 8:441-98.

13. Dyrberg T. Prevalence of diabetic autonomic neuropathy measured by simple bedside tests. Diabetologia 20 1981;1904.

14. M Lakhotia, PKD Shah, R Vyas, S S Jain, A Yadav, M K Parihar. Clinical Dysautonomia in Diabetes Mellitus- A Study with Seven Autonomic Reflex Function Tests. JAPI 1997;45(4).

15. R C Gupta, MD Chittora, A Jain. A study of Autonomic Neuropathy in Diabetes Mellitus in relation to its metabolic control. JAPI 1995:43 (7).

16. Low $\mathrm{P}$ A. Recent advances in the pathogenesis of diabetic neuropathy. Muscle Nerve 1987;121-8.

17. Mackay T. Autonomic neuropathy. Diabetologia 1980;18:4718 .

18. Ramachandran A. Epidemiology of vascular complications in Type II diabetes in Indians" Novo Nordisk update 1999;101. 
19. Fagerberg SE. Diabetic neuropathy, A clinical and histological study in the significance of vascular affections. Acta Med Scand $164 ; 1-80$.

20. Tomlinson DR. Polyols and Myoinositol in diabetic neuropathy", Mayo clinic proceeding 1989;64:1030.

21. Greene D A. Complications; neuropathy, pathogenic considerations. Diabetes Care 1992;15:1902-25.

22. Gupta R C. A study of autonomic neuropathy in diabetes mellitus in relation to its metabolic control. JAPI 1995;(43):464-6.
23. Hilsted J. Pathophysiology in diabetic autonomic neuropathy, cardiovascular, hormonal and metabolic studies". Diabetes 1982;(31):730-6. 